\title{
Higher SARS-CoV-2 viral loads correlated with smaller thyroid volumes on ultrasound among male COVID-19 survivors
}

\author{
David Tak Wai Lui $\mathbb{D}^{1} \cdot$ Matrix Man Him Fung $\mathbb{D}^{2} \cdot$ Keith Wan Hang Chiu $\mathbb{D}^{3} \cdot$ Chi Ho Lee $\mathbb{D}^{1} \cdot$ Wing Sun Chow ${ }^{1}$. \\ Alan Chun Hong Lee ${ }^{1}$ - Anthony Raymond Tam ${ }^{1}$. Polly Pang ${ }^{1}$. Tip Yin Ho ${ }^{1}$. Carol Ho Yi Fong ${ }^{1}$. \\ Connie Hong Nin Loong ${ }^{1}$. Wade Wei Wong ${ }^{2} \cdot$ Cassandra Yuen Yan Lee ${ }^{2} \cdot$ Chun Yiu Law ${ }^{4} \cdot$ Kelvin Kai Wang To $\mathbb{D}^{5}$. \\ Ching Wan $\operatorname{Lam}^{6} \cdot$ Kathryn Choon Beng Tan $\mathbb{D}^{1} \cdot$ Yu Cho Woo ${ }^{1} \cdot$ Ivan Fan Ngai Hung $\mathbb{D}^{1} \cdot$ Karen Siu Ling Lam $\mathbb{D}^{1}$. \\ Brian Hung Hin Lang $\mathbb{D}^{2}$
}

Received: 27 June 2021 / Accepted: 19 August 2021 / Published online: 1 September 2021

(c) The Author(s), under exclusive licence to Springer Science+Business Media, LLC, part of Springer Nature 2021

\begin{abstract}
Purpose Thyroid dysfunction, including thyroiditis, is well recognized in COVID-19 patients. We evaluated thyroid ultrasonographic features among COVID-19 survivors, which are less well known.

Methods Adult COVID-19 survivors without known thyroid disorders who attended dedicated COVID-19 clinic underwent thyroid ultrasonography and assessment of thyroid function and autoimmunity. Adults admitted for acute non-thyroidal surgical problems and negative for COVID-19 were recruited as control. SARS-CoV-2 viral load (VL) was presented as the inverse of cycle threshold values from the real-time reverse transcription-polymerase chain reaction of the respiratory specimen on admission.

Results In total, 79 COVID-19 patients and 44 non-COVID-19 controls were included. All abnormal thyroid function tests during acute COVID-19 recovered upon follow-up. Thyroid ultrasonography was performed at a median of 67 days after acute COVID-19. The median thyroid volume was $9.73 \mathrm{~mL}$ (IQR: 7.87-13.70). In multivariable linear regression, SARSCoV-2 VL on presentation (standardized beta $-0.206, p=0.042$ ) inversely correlated with thyroid volume, in addition to body mass index at the time of ultrasonography $(p<0.001)$. Sex-specific analysis revealed similar results among men but not women. Eleven COVID-19 patients (13.9\%) had ultrasonographic changes suggestive of thyroiditis, comparable to nonCOVID-19 patients $(p=0.375)$. None of these 11 patients had isolated low thyroid-stimulating hormone levels suggestive of thyroiditis at initial admission or the time of ultrasonography.

Conclusions Higher SARS-CoV-2 VL on presentation were associated with smaller thyroid volumes, especially in men. Further research is suggested to investigate this possible direct viral effect of SARS-CoV-2 on the thyroid gland. There was no increased rate of ultrasonographic features suggestive of thyroiditis in COVID-19 survivors.
\end{abstract}

Keywords COVID-19 SARS-CoV-2 $\cdot$ Thyroid gland • Ultrasonography $\cdot$ Thyroiditis $\cdot$ Thyroid function tests

These authors contributed equally: David Tak Wai Lui, Matrix Man Him Fung

Brian Hung Hin Lang

blang@hku.hk

1 Department of Medicine, The University of Hong Kong, Queen Mary Hospital, Hong Kong, China

2 Department of Surgery, The University of Hong Kong, Queen Mary Hospital, Hong Kong, China

3 Department of Diagnostic Radiology, The University of Hong Kong, Hong Kong, China

\section{Introduction}

The initial report of SARS-CoV-2-related thyroiditis in May 2020 has triggered subsequent extensive research into the

4 Division of Chemical Pathology, Queen Mary Hospital, Hong Kong, China

5 Department of Microbiology, The University of Hong Kong, Queen Mary Hospital, Hong Kong, China

6 Department of Pathology, The University of Hong Kong, Hong Kong, China 
interaction between COVID-19 and the thyroid gland [1]. Microscopically, angiotensin-converting enzyme 2 (ACE2) mRNA expression was found in thyroid follicular cells [2]. As ACE2 is the receptor for SARS-CoV-2 entry, this provides a potential mechanism of thyroid damage in COVID19. Biochemically, abnormal thyroid function tests (TFTs) suggestive of thyroiditis have been noted in cohorts of non-severe [3], severe [4] and critical [5] COVID-19 patients. Imaging using thyroid ultrasonography (USG) has revealed features of thyroid damage such as hypoechoic areas and decreased vascularity in both the acute and convalescent phases.

However, thyroid USG has been mainly performed in patients who had clinically overt thyroid dysfunction in acute COVID-19 [6, 7]. Little is known in a broader sense on how the infection may affect the subsequent ultrasonographic features and thyroid volumes for COVID-19 survivors. This information could enhance our understanding of SARS-CoV-2-related thyroid damage beyond clinical features and biochemical abnormalities. Identifying the predictors for these ultrasonographic changes may refine the monitoring strategies for subsequent thyroid dysfunction among COVID-19 survivors. Hence, we embarked on this prospective study of thyroid ultrasonographic assessment of COVID-19 survivors to describe their ultrasonographic features and their respective predictors.

\section{Methods}

This study followed the principles in the Declaration of Helsinki and was approved by the Institutional Review Board of the University of Hong Kong/Hospital Authority Hong Kong West Cluster. All participants gave informed consent before the commencement of the study.

\section{Recruitment of COVID-19 patients}

Adult COVID-19 survivors (age $\geq 18$ years) who attended the dedicated COVID-19 clinic of Queen Mary Hospital between 20 January 2021 and 7 April 2021 (inclusive), and $\geq 30$ days after acute COVID-19, were invited to stay behind after the clinic sessions for thyroid USG. All patients had the presence of SARS-CoV-2 confirmed by reverse transcription-polymerase chain reaction (RT-PCR) from the nasopharyngeal swab (NPS) or deep throat saliva (DTS), using the LightMix SarbecoV E-gene assay (TIB Molbiol, Berlin, Germany), which targeted the envelope protein (E) gene of SARS-CoV-2 as previously described $(3,8)$. Exclusion criteria were (i) history of thyroid, hypothalamic or pituitary disorders; (ii) use of anti-thyroid drugs or thyroid hormone replacement; and (iii) use of medications with potential impact on thyroid function at the time of thyroid function measurements, including systemic steroid, amiodarone, heparin, and dopamine.

\section{Recruitment of non-COVID-19 controls}

Adult patients admitted to the non-intensive care surgical unit of Queen Mary Hospital in the same period for acute non-thyroidal surgical illness, fulfilling the above exclusion criteria and confirmed negative for SARS-CoV-2, were invited for thyroid USG, for the comparison of thyroid volumes and thyroiditis changes with COVID-19 survivors.

\section{Assessment of thyroid function and autoimmunity}

Serum TSH, fT4 and fT3 were measured with immunoassays ADVIA Centaur ${ }^{\circledR}$ TSH3-Ultra, FT4 and FT3 assays, respectively (Siemens Healthcare Diagnostics Inc., Erlangen, Germany). The reference ranges for TSH, fT4 and fT3 were $0.35-4.8 \mathrm{mIU} / \mathrm{L}, 12-23 \mathrm{pmol} / \mathrm{L}$ and $3.2-6.5$ $\mathrm{pmol} / \mathrm{L}$, respectively. Anti-thyroglobulin (anti-Tg) and anti-thyroid peroxidase (anti-TPO) antibody titres were measured with QUANTA Lite ${ }^{\circledR}$ Thyroid $\mathrm{T}$ and TPO enzyme-linked immunosorbent assay, respectively (Inova Diagnostics, San Diego, CA, USA). Positive anti-Tg and anti-TPO was defined by $>100$ units $(3)[8,9]$.

\section{Assessments for COVID-19 patients in acute COVID- 19 and at the time of USG}

Each patient had baseline blood tests taken within $24 \mathrm{~h}$ of admission before starting COVID-19 treatments. TSH, fT4, fT3, anti-Tg and anti-TPO were measured. Basic haematology and biochemistry panel, glycated haemoglobin (HbA1c) and C-reactive protein (CRP) were measured. Elevated CRP was defined by $>0.76 \mathrm{mg} / \mathrm{dL}$. COVID-19related symptoms were evaluated with a standard checklist. Respiratory rate, baseline oxygen saturation by pulse oximetry, and oxygen requirement on admission were captured. Chest $\mathrm{x}$-ray was performed on each patient on admission. Cycle threshold $(\mathrm{Ct})$ values were obtained from the qualitative LightMix SarbecoV E-gene assay (TIB Molbiol, Berlin, Germany) performed on specimens from NPS or DTS (whichever was lower). The Ct value represents the number of cycles required for a gene target or a PCR product to be detected. According to the manufacturer's instructions, samples with $\mathrm{Ct}$ value $<39.0$ will be regarded SARS-CoV-2 detected [10]. While viral loads were not directly measured with a dedicated quantitative RT-PCR assay in this analysis, studies have shown a good correlation between $\mathrm{Ct}$ values and SARS-CoV-2 viral loads $[11,12]$, such that the lower the $\mathrm{Ct}$ values, the higher the viral loads. SARS-CoV-2 viral loads were presented as 
$\frac{1}{S A R S-C o V-2 \text { PCRCt value }}$ in the correlation and multivariable analyses for easier understanding. COVID-19 severity was classified according to the "Chinese Clinical Guidance for COVID-19 Pneumonia Diagnosis and Treatment $\left(7^{\text {th }}\right.$ edition)" published by the Chinese National Health Commission [13]. During the COVID-19 clinic visit, patients were systematically evaluated for symptoms with a standard checklist and had a reassessment chest x-ray, CRP, TFTs and anti-thyroid antibodies. Thyroid USG was performed as described below.

\section{Thyroid USG}

All thyroid USG was performed by the same trained operator, blinded to patients' clinical information, using the same ultrasound scanner (LOGIQ e, GE Healthcare, Wisconsin) equipped with a linear transducer (12L-SC, GE Healthcare, Wisconsin). A standardized reporting protocol was adopted. Both grey-scale and colour Doppler images were interpreted in real-time. Normal appearance of the thyroid on USG was defined as a homogeneous parenchyma hyperechoic to the surrounding strap muscles. (Fig. 1) During USG, features of thyroiditis were searched for, i.e., diffuse reduction or diffuse heterogeneous changes (Fig. 2) in thyroid parenchymal echogenicity [14], diffuse micro-nodular changes [15] and diffuse vascularity changes [16, 17]. Reduction in thyroid parenchymal echogenicity was defined by iso- or hypoechoic appearance with reference to the strap muscles. Diffuse micronodular changes were defined as the finding of micro-nodules (1-6 mm) diffusely distributed throughout the thyroid parenchyma. (Fig. 3) Vascularity of the thyroid parenchyma was assessed with colour Doppler (Fig. 4), with the frequency and gain adjusted to a level with the highest sensitivity and yet the least random noise [17]. Vascularity was graded with the following system: reduced - parenchymal flow is absent or limited to few sub-capsular vessels; normal-prevalent blood flow in main peripheral thyroid arteries and sub-capsular vessels, with scarce parenchymal blood flow with patchy, uneven distribution; moderately increased - clearly increased parenchymal blood flow with patchy distribution; and thyroid inferno - markedly increased blood flow with diffuse homogeneous distribution [17]. Abnormal vascularity was defined as increased or reduced vascularity patterns. Ultrasonographic features of individual macro-nodules (nodules with the shortest dimension $\geq 1 \mathrm{~cm}$ ) were not considered thyroiditis changes. Dimensions of the thyroid lobes (width [W], depth [D] and length [L]) were measured. The volume of each thyroid lobe (V) was calculated by the formula $\mathrm{V}=\mathrm{W} \times \mathrm{D} \times \mathrm{L} \times 0.523$ [18]. Thyroid volume was calculated by the sum of the volume of both lobes. Isthmic volume was included in thyroid volume calculation if the isthmic anteroposterior diameter was $>0.5 \mathrm{~cm} \mathrm{[17].}$

\section{Definitions of comorbidities}

Both active smokers and ex-smokers were considered to have smoking habits. Both chronic drinkers and ex-drinkers were considered to have drinking habits. Body weight and height were measured at the time of thyroid USG. Obesity was defined by an Asian-specific body mass index (BMI) cut-off of $27.5 \mathrm{~kg} / \mathrm{m}^{2}$. Diabetes was defined by a known diagnosis of diabetes or $\mathrm{HbA} 1 \mathrm{c} \geq 6.5 \%$ on admission. Charlson comorbidity index was calculated for each patient.

\section{Statistical analyses}

All statistical analyses were performed with IBM $^{\circledR}$ SPSS $^{\circledR}$ version 26. Two-sided $p$ values $<0.05$ were considered

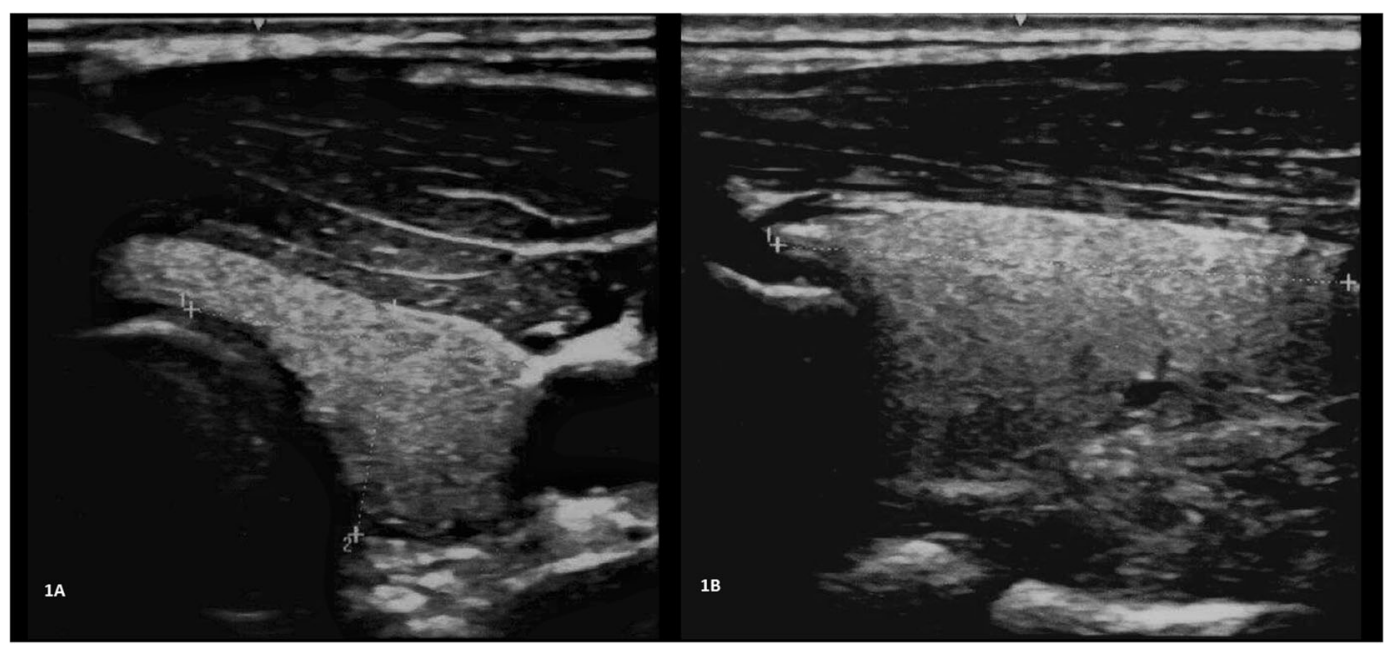

Fig. 1 Ultrasound images of the normal left thyroid lobe in a COVID-19 survivor showing homogeneous parenchymal echogenicity, hyperechoic to surrounding strap muscles. (1A: transverse view, 1B: longitudinal view) 

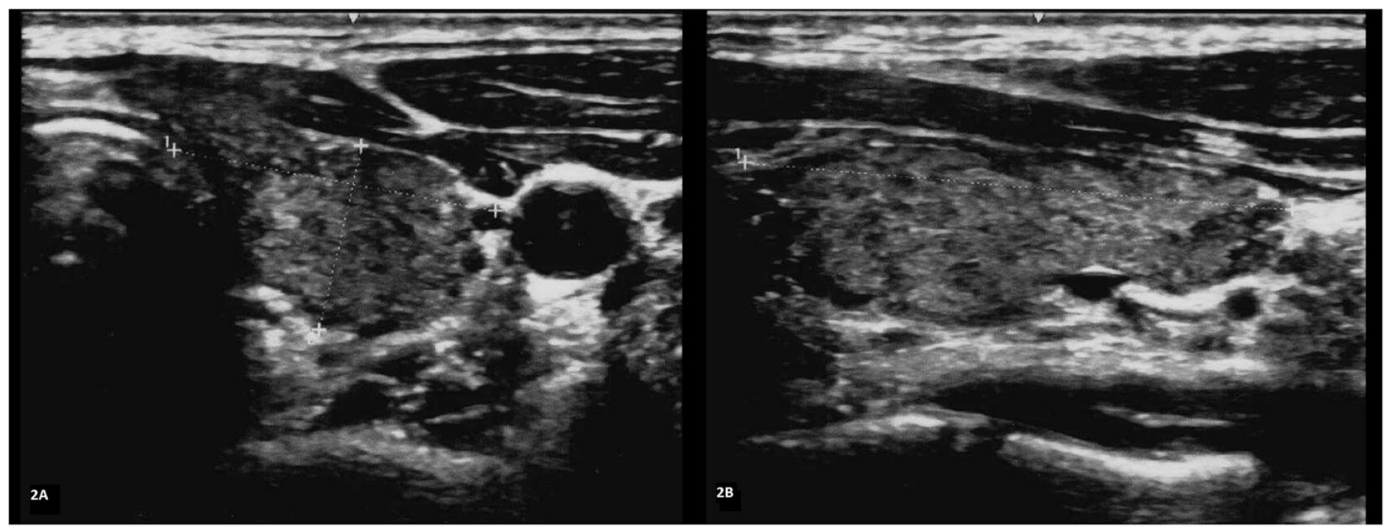

Fig. 2 Ultrasound images of the left thyroid lobe in a COVID-19 survivor showing diffuse heterogeneous echogenicity throughout the thyroid parenchyma. (2A: transverse view, 2B: longitudinal view)
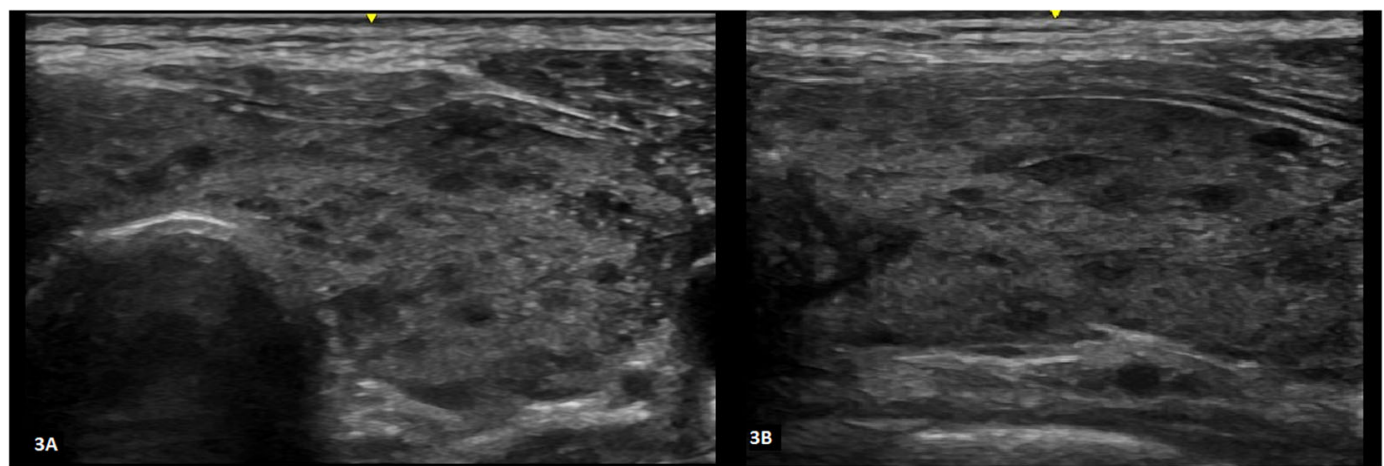

Fig. 3 Ultrasound images of the left thyroid lobe in a COVID-19 survivor showing multiple hypoechoic micro-nodules (1-6 mm) diffusely distributed throughout the thyroid parenchyma. (3A: transverse view, 3B: longitudinal view)

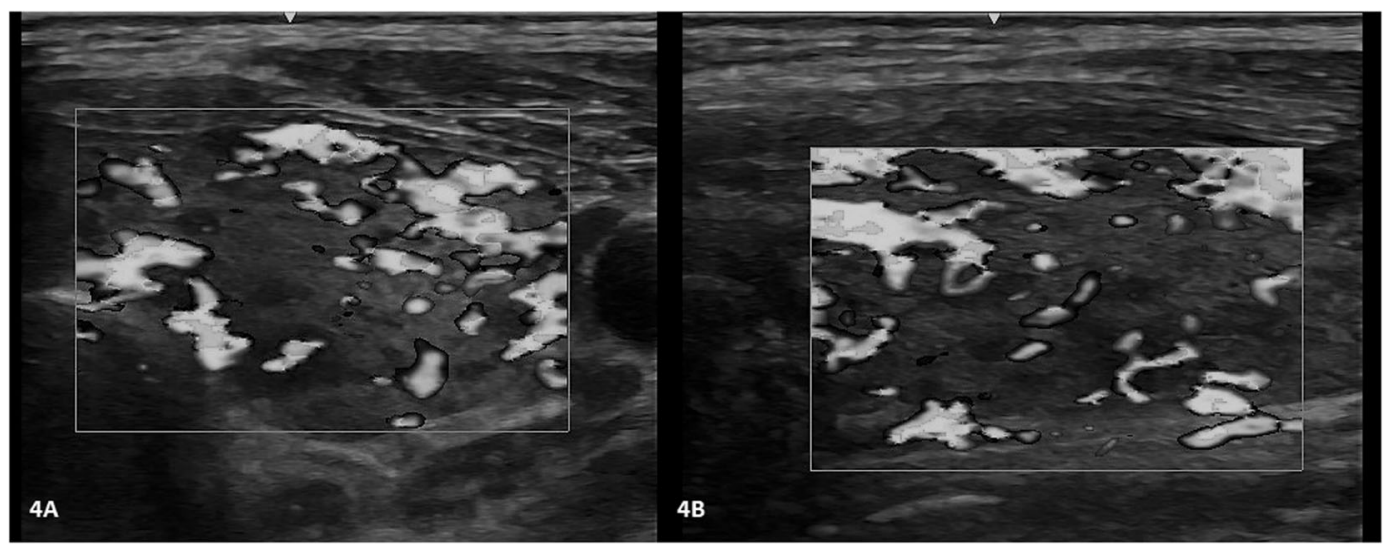

Fig. 4 Colour Doppler images of the left thyroid lobe in a COVID-19 survivor showing clearly increased parenchymal vascularity with patchy distribution. (4A transverse view, $\mathbf{4 B}$ : longitudinal view)

statistically significant. Data were presented as median with interquartile range (IQR) or number with percentage as appropriate. Between-group comparisons were performed with the $t$ test or Mann-Whitney $\mathrm{U}$ test for continuous variables as appropriate, and Chi-square or Fisher's exact tests for categorical variables as appropriate. Pearson correlation was used to study the correlation between continuous variables. Data not normally distributed were transformed logarithmically before analyses. Multivariable linear regression analysis was used to identify the variables independently associated with thyroid volume. All variables with statistical 
significance in the univariate analysis $(p<0.05)$ were included in the multivariable regression analysis.

\section{Results}

In total, 79 COVID-19 survivors responded to the invitation and participated in this study. The clinical characteristics of the COVID-19 survivors are summarized in Table 1. The median age was 48 years (IQR: 36-59), and 54 (68.4\%) were male. The median BMI was $25.2 \mathrm{~kg} / \mathrm{m}^{2}$ (IQR: 22.8-29.4). They were commonly comorbid with hypertension (15.2\%) and diabetes (10.1\%). Regarding their acute COVID-19 illness, most (89.9\%) had a non-severe disease, and only two required intensive care unit admission. Among the 45 patients who had TFTs checked on admission for acute COVID-19, eight were abnormal (5 had isolated low fT3 typical of non-thyroidal illness syndrome, two had isolated low TSH suggestive of thyroiditis, and one had isolated mildly low fT4 of $11 \mathrm{pmol} / \mathrm{L}$ ). None of them had overt thyrotoxicosis or overt hypothyroidism. Upon reassessment of these patients at the dedicated COVID-19 clinic, all had TSH, fT4 and fT3 within normal range. In total, 44 non-COVID-19 controls were recruited (Table 1). The $\%$ male, smoking and drinking habits, thyroid function and antibody positivity were comparable to the COVID-19 survivors. However, non-COVID controls were older with lower BMI and more comorbidities.

Among the COVID-19 survivors, thyroid USG was performed at a median of 67 days (IQR: 41-108) after acute COVID-19. Among the 61 patients with TFTs checked upon thyroid ultrasonographic assessment, all were normal. CRP levels in all COVID-19 patients had normalized except for one. The median thyroid volume was $9.73 \mathrm{~mL}$ (IQR: 7.87-13.70).

\section{Variables associated with thyroid volume among COVID-19 survivors}

Among the continuous variables, only BMI and SARSCoV-2 viral load on presentation correlated with the thyroid volume (Table 2). Thyroid volumes were significantly higher in men than in women (male: $10.9 \mathrm{~mL}$ [IQR: 8.5-14.2] vs female: $8.2 \mathrm{~mL}$ [IQR: $6.8-11.8$ ], $p=0.016$ ). Among other categorical variables, thyroid volumes were not different when classified according to comorbidities, smoking $(p=0.199)$ and drinking $(p=0.113)$ habits, clinical severity of acute COVID-19 $(p=0.187)$, treatment exposure during acute COVID-19 $(p=0.448)$, CRP elevation during acute COVID-19 $(p=0.993)$ or at the time of thyroid USG $(p=0.139)$, and anti-TPO/Tg positivity during acute COVID-19 or at the time of thyroid USG. Thyroid volumes did not differ among COVID-19 survivors who did and did not have non-thyroidal illness syndrome, characterized by the presence of low fT3, in the acute illness $(p=0.070)$.

In the multivariable linear regression analysis including BMI, SARS-CoV-2 viral load and sex, BMI (standardized beta $0.386, p<0.001$ ) and SARS-CoV-2 viral load (standardized beta $-0.206, p=0.042$ ) remained to be the independent determinants of thyroid volume, but not sex $(p=0.082)$.

Given the significant difference in thyroid volume between male and female, we repeated the sex-specific analysis of clinical characteristics associated with thyroid volume. In male, only BMI $(r=0.357, p=0.008)$ and SARS-CoV-2 viral load on presentation $(r=-0.326$, $p=0.016)$ showed significant correlations with thyroid volume in univariate analysis. In the multivariable linear regression analysis, both BMI (standardized beta 0.300 , $p=0.024)$ and SARS-CoV-2 viral load (standardized beta $-0.260, p=0.049)$ remained independent factors associated with thyroid volume. On the other hand, in female, only BMI showed a significant correlation with thyroid volume (standardized beta $0.535, p=0.007$ ).

\section{Ultrasonographic changes suggestive of thyroiditis among COVID-19 survivors}

Ultrasonographic changes suggestive of thyroiditis were observed in 11 patients (13.9\%): 5 had heterogeneous echogenicity, 6 had abnormal vascularity ( 2 increased and 4 decreased), and 3 had micro-nodulation. None of the patients was noted to have hypoechoic thyroid parenchyma. For patients with increased thyroid parenchymal vascularity, none had a thyroid inferno pattern. In total, 15 patients had macro-nodules in the thyroid. Among those with TFTs checked, none of these patients with ultrasonographic changes suggestive of thyroiditis had isolated low TSH suggestive of thyroiditis at the initial admission or at the time of thyroid USG. The clinical profiles of patients with ultrasonographic changes suggestive of thyroiditis are summarized in Table 3.

Comparison between the patients with and without ultrasonographic changes suggestive of thyroiditis revealed that all the clinical characteristics in acute COVID-19 and at the time of thyroid USG were comparable. (Data not shown).

\section{Comparison of ultrasonographic features with non- COVID-19 patients}

The median thyroid volume of the non-COVID-19 patients was $10.2 \mathrm{~mL}$ (IQR: $8.5-13.5$ ), comparable to that of our 
Table 1 Clinical characteristics of the COVID-19 survivors and nonCOVID-19 controls

\begin{tabular}{lclr}
\hline & $\begin{array}{l}\text { COVID-19 } \\
\text { survivors } \\
(n=79)\end{array}$ & $\begin{array}{l}\text { Non-COVID- } \\
19 \text { controls } \\
(n=44)\end{array}$ & $P$ value \\
\hline Age (years) & $48(36-59)$ & $65(50-73)$ & $<0.001$ \\
Male & $54(68.4 \%)$ & $25(56.8 \%)$ & 0.201 \\
Smoking & $11(13.9 \%)$ & $4(9.1 \%)$ & 0.570 \\
Drinking & $18(22.8 \%)$ & $6(13.6 \%)$ & 0.220 \\
BMI $\left(\mathrm{kg} / \mathrm{m}^{2}\right)$ & 25.2 & 23.1 & 0.010 \\
Charlson comorbidity & $(22.8-29.4)$ & $(20.7-25.3)$ & \\
0 & $65(82.2 \%)$ & $17(38.6 \%)$ & \\
$\geq 1$ & $14(17.8 \%)$ & $27(61.4 \%)$ & \\
Obesity & $29(36.7 \%)$ & $6(13.6 \%)$ & 0.007 \\
Hypertension & $12(15.2 \%)$ & $15(34.1 \%)$ & 0.015 \\
Diabetes & $8(10.1 \%)$ & $10(22.7 \%)$ & 0.058
\end{tabular}

Initial admission for COVID-19

Symptomatic of $\quad 65(82.2 \%) \quad$ N/A

COVID-19

Baseline clinical severity

\begin{tabular}{lllll} 
Mild & $51(64.6 \%)$ & N/A & $1(1.3 \%)$ & N/A \\
Moderate & $27(34.2 \%)$ & & $\begin{array}{l}\text { Elevated CRP at } \\
\text { thyroid USG }\end{array}$ & \\
\hline
\end{tabular}

Table 1 (continued)

\begin{tabular}{|c|c|c|c|}
\hline & $\begin{array}{l}\text { COVID-19 } \\
\text { survivors } \\
(n=79)\end{array}$ & $\begin{array}{l}\text { Non-COVID- } \\
19 \text { controls } \\
(n=44)\end{array}$ & $P$ value \\
\hline $\begin{array}{l}\text { Heterogeneous } \\
\text { echogenicity }\end{array}$ & $5(6.3 \%)$ & $2(4.5 \%)$ & 0.999 \\
\hline Abnormal vascularity & $6(7.6 \%)$ & $1(2.3 \%)$ & 0.420 \\
\hline Micronodular pattern & $3(3.8 \%)$ & $0(0 \%)$ & 0.552 \\
\hline $\begin{array}{l}\text { Any of the suggestive } \\
\text { thyroiditis changes }\end{array}$ & $11(13.9 \%)$ & $3(6.8 \%)$ & 0.37 \\
\hline $\begin{array}{l}\text { TSH at thyroid USG } \\
(\mathrm{mIU} / \mathrm{L})\end{array}$ & $\begin{array}{l}1.40 \\
(0.95-1.90)^{\mathrm{b}}\end{array}$ & $\begin{array}{l}1.15 \\
(0.68-1.85)\end{array}$ & 0.185 \\
\hline $\begin{array}{l}\text { fT4 at thyroid USG } \\
(\mathrm{pmol} / \mathrm{L})\end{array}$ & $\begin{array}{l}17.0 \\
(15.0-19.0)^{\mathrm{b}}\end{array}$ & $\begin{array}{l}17.5 \\
(15.0-19.0)\end{array}$ & 0.617 \\
\hline $\begin{array}{l}\text { fT3 at thyroid USG } \\
(\mathrm{pmol} / \mathrm{L})\end{array}$ & $4.9(4.6-5.3)^{\mathrm{b}}$ & N/A & \\
\hline $\begin{array}{l}\text { Abnormal TFTs at } \\
\text { thyroid USG }\end{array}$ & $0 / 61(0 \%)$ & $3 / 44(6.8 \%)$ & 0.071 \\
\hline $\begin{array}{l}\text { Anti-TPO positivity at } \\
\text { thyroid USG }\end{array}$ & $7 / 54(13.0 \%)$ & 4/30 (13.3\%) & 0.999 \\
\hline $\begin{array}{l}\text { Anti-TG positivity at } \\
\text { thyroid USG }\end{array}$ & $5 / 54(9.3 \%)$ & $3 / 30(10.0 \%)$ & 0.999 \\
\hline $\begin{array}{l}\text { Elevated CRP at } \\
\text { thyroid USG }\end{array}$ & $1(1.3 \%)$ & N/A & \\
\hline
\end{tabular}

Baseline SARS-CoV-2 22.40

PCR Ct value (17.0-28.49)

Baseline TSH (mIU/L) $\quad 1.00$ $(0.71-1.70)^{\mathrm{a}}$

Baseline fT4 (pmol/L) 17.0 $(15.0-18.8)^{\mathrm{a}}$

Baseline fT3 (pmol/L) $4.0(3.9-4.3)^{\mathrm{a}}$

Abnormal TFTs $\quad 8 / 45(17.9 \%)$

Anti-TPO positivity $\quad 6 / 43(14.0 \%)$

Anti-TG positivity $\quad 7 / 43(16.3 \%)$

Elevated CRP $\quad 43(54.4 \%)$

Length of stay (days) 9 (6-13)

Exposure to COVID-19 41 (51.9\%)

treatments

$\begin{array}{ll}\text { Remdesivir } & 29(36.7 \%) \\ \text { Interferon beta-1b } & 27(34.2 \%) \\ \text { Dexamethasone } & 11(13.9 \%) \\ \text { Ribavirin } & 8(10.1 \%) \\ \text { Clofazimine } & 4(5.1 \%) \\ \text { Lopinavir/ritonavir } & 2(2.5 \%) \\ \text { Tocilizumab } & 1(1.3 \%) \\ \text { Intensive care unit } & 2(2.5 \%)\end{array}$

admission

Thyroid USG

Interval from acute $67(42-108)$

COVID-19 (days)

Thyroid volume $(\mathrm{mL}) \quad 9.7(7.9-13.7) \quad 10.2(8.5-13.5) \quad 0.691$

Data are presented as number (percentage) and median (interquartile range) as appropriate

$B M I$ body mass index, $C t$ cycle threshold, TSH thyroid-stimulating hormone, $f T 4$ free thyroxine, $f T 3$ free triiodothyronine, TFTs thyroid function tests, TPO thyroid peroxidase, $T g$ thyroglobulin, $C R P$ C-reactive protein, $U S G$ ultrasonography, $N / A$ not applicable

${ }^{\mathrm{a}} n=45$

$\mathrm{b}_{n}=61$

Table 2 Pearson correlation of clinical parameters with thyroid volume

\begin{tabular}{|c|c|c|}
\hline & Correlation coefficient & $P$ value \\
\hline Age (years) & 0.105 & 0.355 \\
\hline $\operatorname{BMI}\left(\mathrm{kg} / \mathrm{m}^{2}\right)$ & 0.451 & $<0.001$ \\
\hline $\begin{array}{l}\text { Baseline lymphocyte count } \\
\left(\mathrm{x} 10^{9} / \mathrm{L}\right)^{\mathrm{a}}\end{array}$ & -0.040 & 0.726 \\
\hline $\begin{array}{l}\text { SARS-CoV-2 viral load on } \\
\text { presentation }^{\text {a }}\end{array}$ & -0.231 & 0.041 \\
\hline Interval from admission (days) ${ }^{\mathrm{a}}$ & -0.082 & 0.475 \\
\hline Baseline TSH $(\mathrm{mIU} / \mathrm{L})^{\mathrm{a}}$ & 0.057 & 0.109 \\
\hline TSH upon follow-up (mIU/L) ${ }^{\mathrm{a}}$ & -0.224 & 0.080 \\
\hline
\end{tabular}

Values reaching statistical significance are in bold

${ }^{a}$ Logarithmically transformed before analysis

$B M I$ body mass index, $C t$ cycle threshold, TSH thyroid-stimulating hormone

COVID-19 patients $(p=0.691)$. Regarding the thyroiditis changes, diffuse reduction in thyroid parenchymal echogenicity was observed in 1 patient, heterogeneous echogenicity in 2 patients, abnormal vascularity in 1 patient (reduced 


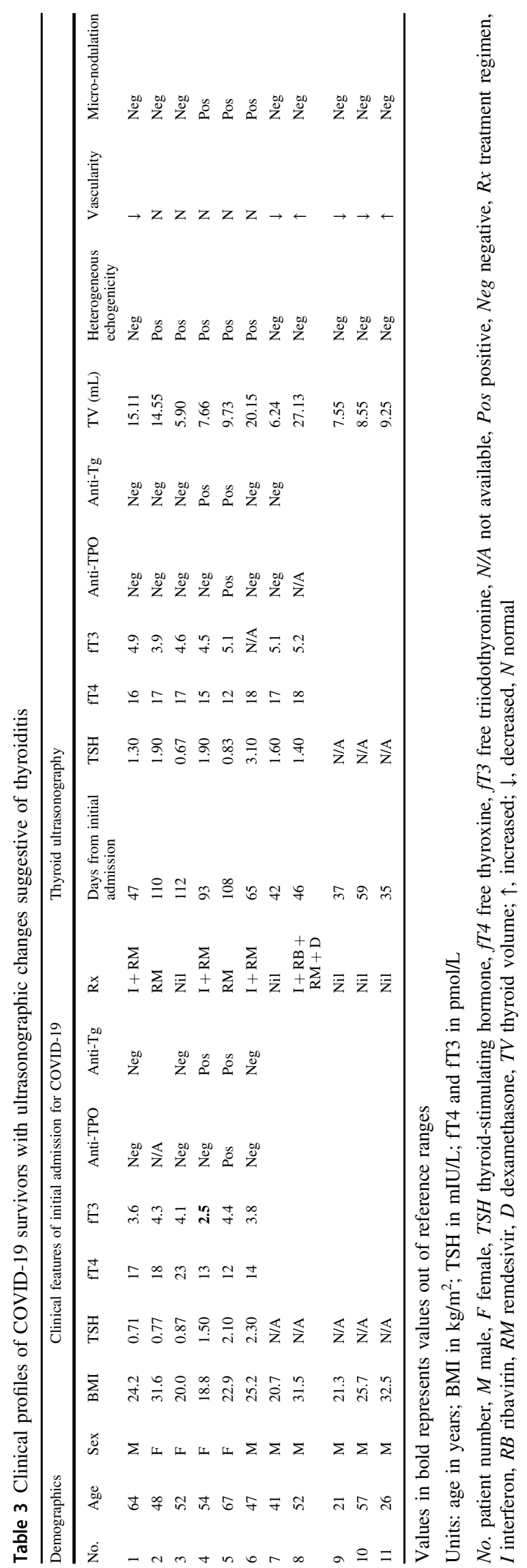

vascularity), and none had a diffuse micro-nodular pattern. Altogether, 3 patients $(6.8 \%)$ had ultrasonographic features compatible with thyroiditis, statistically not significantly different from the COVID-19 survivors $(p=0.375)$.

\section{Discussion}

To date, this is the first study of thyroid ultrasonographic features among COVID-19 survivors in general, in contrast to existing studies focusing on those having clinically overt thyroid dysfunction in acute COVID-19. Interestingly, higher SARS-CoV-2 viral loads on presentation were associated with smaller thyroid volumes among the survivors. This finding encourages longitudinal follow-up to clarify a possible direct viral effect of thyroid atrophy. We observed $13.9 \%$ of the COVID-19 survivors having ultrasonographic features suggestive of thyroiditis, comparable to that among non-COVID-19 patients.

Consistent with existing studies [19], the biochemical profile of our cohort confirmed that the abnormal TFTs in acute COVID-19 largely recovered during convalescence. Most of these abnormal TFTs in the acute phase represent non-thyroidal illness syndrome, characterized by isolated low fT3, due to the COVID-19-related inflammation and the associated cytokine release [3, 20]. Although most COVID19 patients' abnormal TFTs resolved upon short-term follow-up, the long-term sequelae of COVID-19 on the thyroid remained to be elucidated. Evaluation of ultrasonographic features of the thyroid glands of COVID-19 survivors may shed light on this issue.

The key finding of our study was the independent correlation of higher SARS-CoV-2 viral loads on presentation with smaller thyroid volumes in the convalescence phase. Limited reports on post-mortem examination of COVID-19 patients did not reveal significant structural abnormalities in the thyroid, except for reports of inflammatory infiltrates in the interstitium, though these were not dedicated studies of the thyroid [21]. Further insights can be obtained from detailed post-mortem examinations focusing on the thyroid in SARS-CoV-1 infected patients reporting extensive damage of parafollicular and follicular epithelial cells and destroyed epithelial cells, leading to the collapse and distortion of follicular architecture [22]. In the acute phase of thyroiditis associated with COVID-19, the thyroid volume was reported to be increased or normal [21, 23, 24], likely explained by the acute inflammation in the thyroid [25]. In the convalescence phase after acute COVID-19, however, the thyroid volume might be reduced because of the potential collapse and distortion of the thyroid follicular micro-structure extrapolating from changes reported with SARS-CoV-1 [22]. Indeed, we have previously reported that COVID-19 patients with isolated low TSH suggestive 
of thyroiditis had higher SARS-CoV-2 viral loads [3], suggesting a possible direct viral effect on the thyroid. The latest report of autopsy cases showed the presence of staining for SARS-CoV-2 nucleocapsid antigen in the epithelial cells lining thyroid follicles in patients with COVID19 confirmed by RT-PCR, further supporting a direct viral effect on the thyroid in COVID-19 [26]. The expression of ACE2, the receptor for SARS-CoV-2 entry, in the thyroid provides a potential mechanism for thyroid damage. Of note, in our previous study [3] and this current study, elevated CRP, which reflects systemic inflammation, was not associated with isolated low TSH or the thyroid volume. Furthermore, in this study, non-thyroidal illness syndrome during acute COVID-19 did not influence the thyroid volume. Hence, our novel observation of the association between the SARS-CoV-2 viral load and the thyroid volume further supports a potential direct viral atrophic effect on the thyroid, rather than systemic inflammation or non-thyroidal illness syndrome. Nonetheless, further longitudinal follow-up studies are necessary to establish a causal relationship.

Sex-specific analysis revealed another interesting finding that the inverse correlation between SARS-CoV-2 viral loads and thyroid volume remained significant in men but not in women. Many cases of COVID-19-related thyroid dysfunction reported appeared to demonstrate female preponderance. To date, a total of 13 cases of SARS-CoV-2related subacute thyroiditis have been reported, predominantly affecting female [27]. An earlier review included the description of 3 women and 1 man (aged between 18 and 69 years) having SARS-CoV-2-related subacute thyroiditis [28], characterized by the typical presentation of fever and neck pain, together with elevated erythrocyte sedimentation rate and CRP, negative antithyroid antibodies, and typical ultrasonographic and thyroid scan features. A recent case report described a 57-year-old woman with typical presentation of subacute thyroiditis, associated with positive anti-TPO and anti-Tg as well [24]. Moreover, a recent review discussed the potential of SARSCoV-2 in inducing thyroid autoimmunity, hence, potentially Graves' disease, which again predominantly affects women [29]. In contrast, our study focuses on SARS-CoV-2 associated thyroiditis, without the typical presentation of fever and neck pain. Hence, in some way, the phenotype resembles the entity termed 'SARS-CoV-2-related atypical thyroiditis' described by Muller et al. [5], characterized by the absence of thyroidal pain and swelling despite bearing similar ultrasonographic and thyroid scan features as the typical subacute thyroiditis. In typical subacute thyroiditis, there is a pathognomonic infiltration of giant cells with swelling of thyroid follicles, leading to stretching of the thyroid capsule and causing neck pain. On the contrary, in SARS-CoV-2-related atypical thyroiditis, in view of associated lymphopenia, giant cells might not form and thyroid cells might be damaged by apoptosis as observed in SARS-CoV-1 [22]. ACE2 provides a pathogenic link for the SARS-CoV-2-associated thyroiditis. ACE2 is more highly expressed in thyroid cells than in lung cells, and in women such expression negatively correlates with signatures of immune cell enrichment [30]. This expression profile might explain why severe COVID-19 and the SARSCoV-2-related atypical thyroiditis more often occur in men, in contrast to the female preponderance observed in typical subacute thyroiditis.

Although we observed a rate of $13.9 \%$ of ultrasonographic features suggestive of thyroiditis in this cohort of COVID-19 survivors, it was comparable to that observed among non-COVID-19 patients. Indeed, this rate is also similar to the background rate of abnormal thyroid ultrasonographic echogenicity reported in population studies (around 10\%) [31]. Existing studies have focused on the ultrasonographic features of COVID-19 survivors who had symptomatic SARS-CoV-2-related thyroiditis in acute illness [7]. In contrast, our study attempted to identify any subclinical ultrasonographic suggestion of thyroid parenchymal damage, as these abnormalities could predict subsequent thyroid dysfunction [32]. Results from our study do not suggest additional benefits of routine thyroid ultrasonographic assessment among COVID-19 patients without clinically overt thyroiditis.

The strengths of our study included the following. First, the spectrum of clinical severity of acute COVID-19 represented that of the global situation, with most having non-severe acute COVID-19 [33]. Hence, our results could be generalized to COVID-19 patients at large. Moreover, we evaluated the potential predictors of ultrasonographic features in COVID-19 survivors among a diverse panel of variables (demographics, SARS-CoV-2 viral load and clinical features in acute COVID-19, inflammatory markers, and thyroid function and autoimmunity). However, our study results should be interpreted bearing certain limitations. First, as all COVID-19 patients were treated in an isolation facility in acute COVID-19, thyroid USG was not performed at the time of presentation, such that there were no baseline ultrasonographic features and thyroid volumes for comparison. Nonetheless, non-COVID-19 patients admitted for acute non-thyroidal surgical problems had been recruited for comparison. Second, Ct values obtained from the qualitative RT-PCR assay of SARS-CoV-2 were used as the surrogate of the viral load, which should have preferably been measured directly with a quantitative RT-PCR assay. Last but not least, selection bias was possible because not all COVID-19 survivors agreed to stay behind after clinic sessions for thyroid USG. Participants in this study were younger (48 years [IQR: $36-59]$ vs 57 years [IQR: 42-66] 
vs $p<0.001)$ and more likely male $(68.4 \%$ vs $42.1 \%, p<$ $0.001)$ than those who did not participate. Otherwise, the severity of acute COVID-19 $(p=0.541)$, initial SARSCoV-2 viral load $(p=0.782)$, and TSH levels both at the baseline $(p=0.571)$ and at the time of USG $(p=0.496)$ were all comparable.

\section{Conclusions}

SARS-CoV-2 viral load in acute COVID-19 inversely correlated with the thyroid volume, especially in men, suggesting a possible direct viral effect of SARS-CoV-2 on the thyroid. Follow-up studies are warranted to establish this causal relationship. On the other hand, ultrasonographic features suggestive of thyroiditis occurred in $13.9 \%$ of COVID-19 survivors, not in excess compared to nonCOVID-19 patients and the general population. Hence, routine thyroid ultrasonographic assessment does not appear to help identify subclinical thyroid parenchymal changes beyond the biochemical assessment of thyroid function in COVID-19 patients.

\section{Data availability}

Datasets generated during and/or analyzed during the current study are not publicly available but are available from the corresponding author on reasonable request.

Author contributions D.T.W.L. and M.M.H.F. wrote the manuscript. D.T.W.L, M.M.H.F., K.W.H.C., C.H.L., W.S.C., A.C.H.L., A.R.T., P.P., T.Y.H., W.W.W., C.Y.Y.L., C.H.N.L. and C.Y.L. researched the data. D.T.W.L. and C.H.Y.F. performed statistical analyses. C.H.L., W.S.C., A.C.H.L., K.K.W.T., C.W.L., K.C.B.T., Y.C.W., I.F.N.H., K.S.L.L., B.L. critically reviewed and edited the manuscript. B.L. initiated and supervised the study, is the guarantor of this work, has full access to all the data in the study and takes responsibility for the integrity of the data and the accuracy of the data analysis.

\section{Compliance with ethical standards}

Conflict of interest The authors declare no competing interests.

Publisher's note Springer Nature remains neutral with regard to jurisdictional claims in published maps and institutional affiliations.

\section{References}

1. A. Brancatella, D. Ricci, N. Viola, D. Sgrò, F. Santini, F. Latrofa, Subacute thyroiditis after Sars-COV-2 infection. J. Clin. Endocrinol. Metab. 105(7), dgaa276 (2020)

2. M. Rotondi, F. Coperchini, G. Ricci, M. Denegri, L. Croce, S.T. Ngnitejeu et al. Detection of SARS-COV-2 receptor ACE-2 mRNA in thyroid cells: a clue for COVID-19-related subacute thyroiditis. J. Endocrinol. Invest 44(5), 1085-1090 (2021)
3. D.T.W. Lui, C.H. Lee, W.S. Chow, A.C.H. Lee, A.R. Tam, C.H.Y. Fong, C.Y. Law, E.K.H. Leung, K.K.W. To, K.C.B. Tan, Y.C. Woo, C.W. Lam, I.F.N. Hung, K.S.L. Lam, Thyroid dysfunction in relation to immune profile, disease status, and outcome in 191 patients with COVID-19. J. Clin. Endocrinol. Metab. 106(2), e926-e935 (2021)

4. A. Lania, M.T. Sandri, M. Cellini, M. Mirani, E. Lavezzi, G. Mazziotti, Thyrotoxicosis in patients with COVID-19: the THYRCOV study. Eur. J. Endocrinol. 183(4), 381-387 (2020)

5. I. Muller, D. Cannavaro, D. Dazzi, D. Covelli, G. Mantovani, A. Muscatello et al. SARS-CoV-2-related atypical thyroiditis. Lancet Diabetes Endocrinol. 66(20), 2019-2021 (2020)

6. P. Caron, Thyroiditis and SARS-CoV-2 pandemic: a review. Endocrine 72(2), 326-331 (2021)

7. A. Pizzocaro, P. Colombo, W. Vena, S. Ariano, P. Magnoni, F. Reggiani, G. Favacchio, M. Mirani, E. Lavezzi, A. Voza, M. Calatroni, G. Mazziotti, A. Lania, Humanitas COVID-19 task force. Outcome of Sars-COV-2-related thyrotoxicosis in survivors of Covid-19: a prospective study. Endocrine 73(2), 255-260 (2021)

8. D.T.W. Lui, C.H. Lee, W.S. Chow, A.C.H. Lee, A.R. Tam, C.H. Y. Fong, C.Y. Law, E.K.H. Leung, K.K.W. To, K.C.B. Tan, Y.C. Woo, C.W. Lam, I.F.N. Hung, K.S.L. Lam, Role of non-thyroidal illness syndrome in predicting adverse outcomes in COVID-19 patients predominantly of mild-to-moderate severity. Clin. Endocrinol. (2021). https://doi.org/10.1111/cen.14476

9. D.T.W. Lui, C.H. Lee, W.S. Chow, A.C.H. Lee, A.R. Tam, C.H. Y. Fong, C.Y. Law, E.K.H. Leung, K.K.W. To, K.C.B. Tan, Y.C. Woo, C.W. Lam, I.F.N. Hung, K.S.L. Lam, Insights from a prospective follow-up of thyroid function and autoimmunity among COVID-19 survivors. Endocrinol. Metab. 36(3), 582-589 (2021)

10. J.H.K. Chen, C.C.Y. Yip, J.F.W. Chan, R.W.S. Poon, K.K.W. To, K.H. Chan, et al. Clinical performance of the luminex nxtag cov extended panel for sars-cov-2 detection in nasopharyngeal specimens from covid-19 patients in Hong Kong. J. Clin. Microbiol. 58, e00936-20 (2020)

11. F. Yu, L. Yan, N. Wang, S. Yang, L. Wang, Y. Tang et al. Quantitative detection and viral load analysis of SARS-CoV-2 in infected patients. Clin. Infect. Dis. 71(15), 793-798 (2020)

12. M.R. Tom, M.J. Mina, To interpret the SARS-CoV-2 test, consider the cycle threshold value. Clin. Infect. Dis. 71(16), 2252-2254 (2020)

13. Chinese Clinical Guidance for COVID-19 Pneumonia Diagnosis and Treatment (7th edition) [Internet]. [cited 2020 Aug 23]. Available from: http://kjfy.meetingchina.org/msite/news/show/cn/ 3337.html

14. D.Y. Shin, E.-K. Kim, E.J. Lee, Role of ultrasonography in outcome prediction in subclinical hypothyroid patients treated with levothyroxine. Endocr. J. 57(1), 15-22 (2010)

15. H.C. Yeh, W. Futterweit, P. Gilbert, Micronodulation: ultrasonographic sign of Hashimoto thyroiditis. J. Ultrasound Med. 15 (12), 813-819 (1996)

16. L.F. Alexander, N.J. Patel, M.P. Caserta, M.L. Robbin, Thyroid ultrasound: diffuse and nodular disease. Radio. Clin. North Am. 58(6), 1041-1057 (2020)

17. T. Rago, V. Cantisani, F. Ianni, L. Chiovato, R. Garberoglio, C. Durante, et al., Thyroid ultrasonography reporting: consensus of Italian Thyroid Association (AIT), Italian Society of Endocrinology (SIE), Italian Society of Ultrasonography in Medicine and Biology (SIUMB) and Ultrasound Chapter of Italian Society of Medical Radiology. J. Endocrinol. Invest. 41(12), 1435-1443 (2018).

18. M. Dighe, R. Barr, J. Bojunga, V. Cantisani, M.C. Chammas, D. Cosgrove et al. Thyroid ultrasound: state of the art part 1 - Thyroid ultrasound reporting and diffuse thyroid diseases. Med. Ultrason 19(1), 79-93 (2017) 
19. B. Khoo, T. Tan, S.A. Clarke, E.G. Mills, B. Patel, M. Modi et al. Thyroid function before, during, and after COVID-19. J. Clin. Endocrinol. Metab. 106(2), e803-e811 (2021)

20. L. Croce, D. Gangemi, G. Ancona, F. Liboà, G. Bendotti, L. Minelli et al. The cytokine storm and thyroid hormone changes in COVID-19. J. Endocrinol. Invest. 44(5), 891-904 (2021)

21. W. Chen, Y. Tian, Z. Li, J. Zhu, T. Wei, J. Lei, Potential interaction between SARS-CoV-2 and thyroid: a review. Endocrinology 162(3), bqab004 (2021)

22. L. Wei, S. Sun, C.-H. Xu, J. Zhang, Y. Xu, H. Zhu et al. Pathology of the thyroid in severe acute respiratory syndrome. Hum. Pathol. 38(1), 95-102 (2007)

23. L. Davoodi, Z. Oladi, H. Jafarpour, Z. Zakariaei, E. Soleymani, A. Razavi, A 33-year-old man with COVID-19 presented with subacute thyroiditis: a rare case report and literature review. N. Microbes N. Infect. 41(May), 100871 (2021)

24. Dworakowska D., Morley S., Mulholland N., Grossman A.B. COVID-19-related thyroiditis: a novel disease entity? Clin. Endocrinol. (2021):https://doi.org/10.1111/cen.14453

25. L.H. Duntas, J. Jonklaas, COVID-19 and thyroid diseases: a bidirectional impact. J. Endocr. Soc. 5(8 Aug), bvab076 (2021)

26. Poma A.M., Bonuccelli D., Giannini R., Macerola E., Vignali P., Ugolini C., Torregrossa L., Proietti A., Pistello M., Basolo A., Santini F., Toniolo A., Basolo F. COVID-19 autopsy cases: detection of virus in endocrine tissues. J. Endocrinol. Invest. (2021):1-6. 10.1007/s40618-021-01628-y
27. H. Inaba, T. Aizawa, Coronavirus disease 2019 and the thyroidprogress and perspectives. Front Endocrinol. (Lausanne) 12, 708333 (2021). https://doi.org/10.3389/fendo.2021.708333.

28. G. Speer, P. Somogyi, Thyroid complications of SARS and coronavirus disease 2019 (COVID-19). Endocr. J. 68(2), 129-136 (2021)

29. A.K. Murugan, A.S. Alzahrani, SARS-CoV-2 plays a pivotal role in inducing hyperthyroidism of Graves' disease. Endocrine 73(2), 243-254 (2021)

30. M.Y. Li, L. Li, Y. Zhang, X.S. Wang, Expression of the SARSCoV-2 cell receptor gene ACE2 in a wide variety of human tissues. Infect. Dis. Poverty 9(1), 45 (2020). https://doi.org/10.1186/ s40249-020-00662-x

31. P. Vejbjerg, N. Knudsen, H. Perrild, P. Laurberg, I.B. Pedersen, L. B. Rasmussen et al. The association between hypoechogenicity or irregular echo pattern at thyroid ultrasonography and thyroid function in the general population. Eur. J. Endocrinol. 155(4), 547-552 (2006)

32. T. Rago, L. Chiovato, L. Grasso, A. Pinchera, P. Vitti, Thyroid ultrasonography as a tool for detecting thyroid autoimmune diseases and predicting thyroid dsfunction in apparently healthy subjects. J. Endocrinol. Invest. 24(10), 763-769 (2001)

33. Z. Wu, J.M. McGoogan, Characteristics of and important lessons from the coronavirus disease 2019 (COVID-19) outbreak in China: summary of a report of 72,314 cases from the Chinese center for disease control and prevention. JAMA 323(13), 1239-1242 (2020) 